\title{
Evidence of Illegitimate Recombination Between Two Pasteurellaceae Plasmids Resulting in a Novel Multi-Resistance Replicon, pM3362MDR, in Actinobacillus pleuropneumoniae
}

\author{
Yinghui Lit,2t, Giarlã Cunha da Silva ${ }^{3 \dagger}$, Yanwen $\mathrm{Li}^{1}$, Ciro C. Rossi ${ }^{3}$, \\ Roberto Fernandez Crespo', Susanna M. Williamson ${ }^{4}$, Paul R. Langford', \\ Denise Mara Soares Bazzolli ${ }^{3}$ and Janine T. Bossé1*
}

OPEN ACCESS

Edited by:

Katy Jeannot,

Laboratoire Chrono-Environnement,

France

Reviewed by:

Christopher Morton Thomas,

University of Birmingham,

United Kingdom

Bruno Gonzalez-Zorn,

Complutense University of Madrid,

Spain

*Correspondence:

Janine T. Bossé

j.bosse@imperial.ac.uk

†These authors have contributed equally to this work

Specialty section:

This article was submitted to Antimicrobials, Resistance

and Chemotherapy,

a section of the journal

Frontiers in Microbiology

Received: 27 June 2018 Accepted: 28 September 2018

Published: 23 October 2018

Citation:

Li Y, da Silva GC, Li Y, Rossi CC, Fernandez Crespo R, Williamson SM,

Langford PR, Bazzolli DMS and

Bossé JT (2018) Evidence of Illegitimate Recombination Between

Two Pasteurellaceae Plasmids Resulting in a Novel Multi-Resistance Replicon, pM3362MDR,

in Actinobacillus pleuropneumoniae.

Front. Microbiol. 9:2489.

doi: 10.3389/fmicb.2018.02489
${ }^{1}$ Section of Paediatrics, Department of Medicine, Imperial College London, London, United Kingdom, ${ }^{2}$ Shenzhen Center for Disease Control and Prevention, Shenzhen, China, ${ }^{3}$ Laboratório de Genética Molecular de Bactérias, Departamento de Microbiologia, Instituto de Biotecnologia Aplicada à Agropecuária, Universidade Federal de Viçosa, Viçosa, Brazil,

${ }^{4}$ Animal and Plant Health Agency, Addlestone, United Kingdom

Evidence of plasmids carrying the tetracycline resistance gene, tet(B), was found in the previously reported whole genome sequences of 14 United Kingdom, and 4 Brazilian, isolates of Actinobacillus pleuropneumoniae. Isolation and sequencing of selected plasmids, combined with comparative sequence analysis, indicated that the four Brazilian isolates all harbor plasmids that are nearly identical to pB1001, a plasmid previously found in Pasteurella multocida isolates from Spain. Of the United Kingdom isolates, 13/14 harbor plasmids that are (almost) identical to pTetHS016 from Haemophilus parasuis. The remaining United Kingdom isolate, MIDG3362, harbors a 12666 bp plasmid that shares extensive regions of similarity with pOV from P. multocida (which carries bla $\mathrm{ROB}-1_{1}$, sul2, and strAB genes), as well as with pTetHS016. The newly identified multi-resistance plasmid, pM3362MDR, appears to have arisen through illegitimate recombination of pTetHS016 into the stop codon of the truncated strB gene in a pOV-like plasmid. All of the tet(B)-carrying plasmids studied were capable of replicating in Escherichia coli, and predicted origins of replication were identified. A putative origin of transfer (oriT) sequence with similar secondary structure and a nic-site almost identical to that of RP4 was also identified in these plasmids, however, attempts to mobilize them from an RP4-encoding $E$. coli donor strain were not successful, indicating that specific conjugation machinery may be required.

Keywords: plasmids, antimicrobial resistance, tetracycline, respiratory tract, Pasteurellaceae

\section{INTRODUCTION}

Resistance to tetracycline is widespread amongst isolates of Actinobacillus pleuropneumoniae in many countries (Archambault et al., 2012; Vanni et al., 2012; Dayao et al., 2016; El Garch et al., 2016). Despite this, tetracyclines continue to be the most widely used antimicrobial for treatment of respiratory and other diseases in food-producing animals in the United Kingdom and other European countries (Borriello, 2013; Garcia-Migura et al., 2014; European Medicines Agency and European Surveillance of Veterinary Antimicrobial Consumption, 2017). 
Identification of the genes responsible for tetracycline resistance, and an understanding of the mechanisms underlying the spread of these genes, will help inform decisions regarding continued use of this important antimicrobial agent.

Although more than thirty different tetracycline resistance genes have been reported in different bacterial species (Chopra and Roberts, 2001), relatively few have been found in A. pleuropneumoniae, with tet $(\mathrm{B})$ being the most common (Blanco et al., 2006; Dayao et al., 2016; Bossé et al., 2017; Michael et al., 2018). We recently reported that the tet(B) gene was found in the chromosome of $37.5 \%$ of United Kingdom isolates for which whole genome sequences (wgs) were determined, either as part of a large integrative conjugative element (ICEApl1), or as a transposon insertion in the $\operatorname{com} M$ gene (Bossé et al., 2017). A further $14.5 \%$ of the tested isolates had tet(B) genes that appeared to be associated with plasmid sequences (Bossé et al., 2017). Wgs for six Brazilian isolates also indicate the presence of tet(B) associated with plasmid sequences in four of these isolates (Pereira et al., 2015). As small plasmids often appear to be common amongst members of the Pasteurellaceae (Michael et al., 2018), the aim of this study was to identify the tet(B) plasmids present in these sequenced isolates of A. pleuropneumoniae.

\section{MATERIALS AND METHODS}

\section{A. pleuropneumoniae Isolates and Plasmids}

Information regarding the 14 United Kingdom and 4 Brazilian A. pleuropneumoniae isolates and their wgs data
(Table 1) was compiled from details described previously (Pereira et al., 2015; Bossé et al., 2017). Data regarding the tetracycline resistance plasmids identified in this study are also shown (Table 1). Plasmid sequences were initially identified in the draft genome sequences using ResFinder (Zankari et al., 2012) to identify contigs containing the tet(B) gene. BLASTn was then used to identify sequences with the highest identity to each of these contigs. Where it appeared that a single contig matched a known plasmid, the ends of the contigs were analyzed to identify overlapping sequences that allowed closure into a circular plasmid. Where it appeared that the contigs carrying tet(B) represented only partial plasmids, sequences of the plasmids with highest identity were then used to search the wgs using BLASTn to identify other contigs carrying plasmid-related sequences.

Plasmids were extracted from the A. pleuropneumoniae isolates MV780, MIDG2567, MIDG2656, MIDG2658, MIDG3202, MIDG3233, and MIDG3362 using the Qiaprep Spin Miniprep kit (Qiagen) according to the manufacturer's protocol. The correct joining of contigs (i.e., ends of a single, or overlapping multiple contigs, as appropriate) was verified either by inverse PCR to amplify the region between the $5^{\prime}$ and $3^{\prime}$ ends of the tet(B) gene, or by amplification across the predicted contig junctions. The complete sequence of each extracted plasmid was confirmed using a primer walking strategy. Descriptions of all primers used in this study are shown in Table 2. The annotated sequences of plasmids p780, pM2567Tet, pM2656Tet, pM2658Tet, pM3202Tet, pM3233Tet, and pM3362MDR have been deposited in Genbank

TABLE 1 | Actinobacillus pleuropneumoniae isolates carrying tet(B) resistance plasmids.

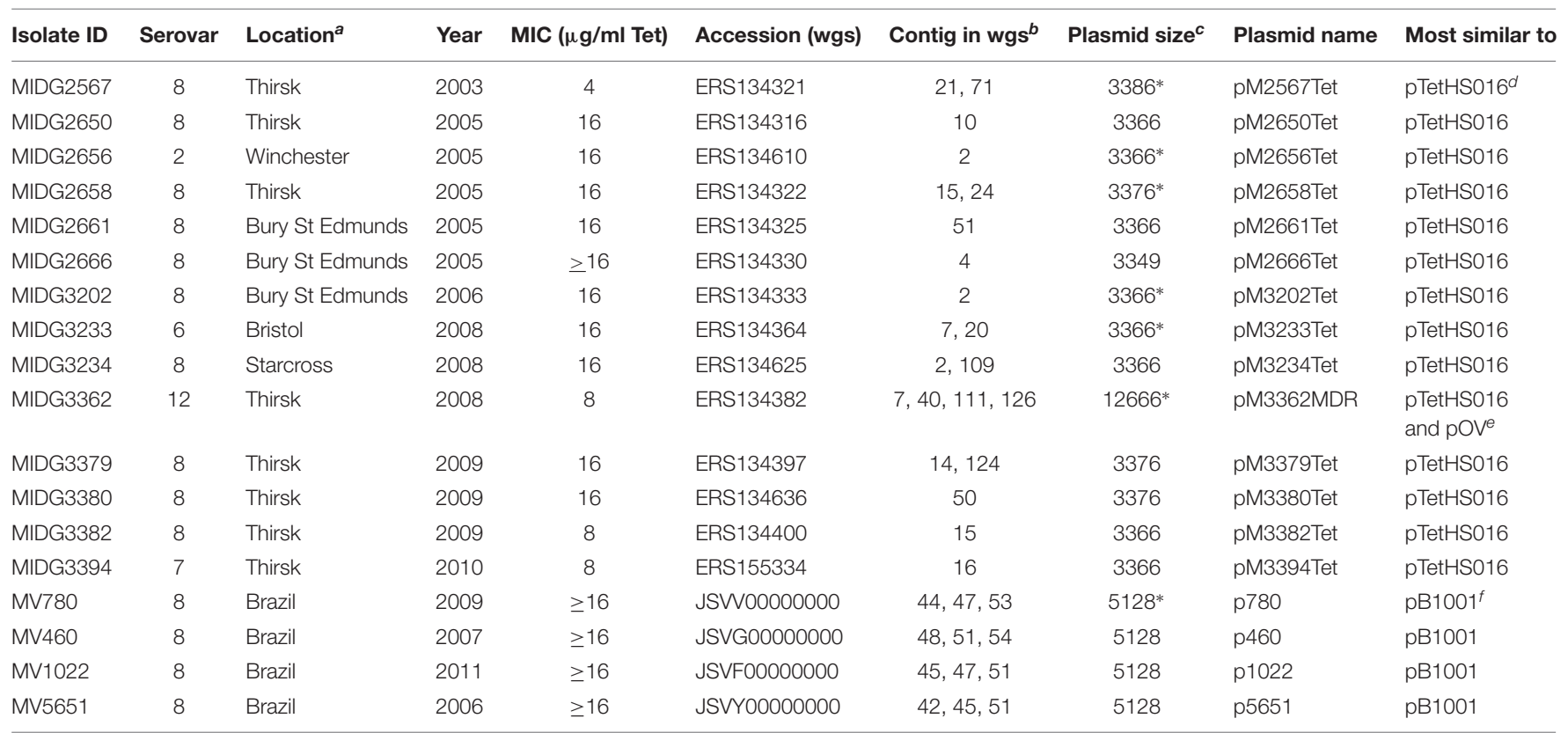

${ }^{a}$ Other than the four Brazilian isolates (Pereira et al., 2015), specific locations are for previously described United Kingdom isolates (Bossé et al., 2017). ${ }^{b}$ Contig number(s) in draft wgs containing plasmid sequences. "Sizes of isolated plasmids confirmed by sequencing are indicated (*), otherwise sizes were predicted bioinformatically. ${ }^{a}$ pTetHSO16 (accession KC818265) is a 3366 bp plasmid from a United Kingdom Haemophilus parasuis isolate (Luan et al., 2013). ${ }^{e}$ pOV (accession NC_019381) is a 13,551 bp plasmid from Pasteurella multocida. ${ }^{f}$ pB1001 (accession EU252517) is a 5128 bp plasmid from P. multocida in Spain. 
TABLE 2 | Primers used in this study.

\begin{tabular}{|c|c|c|}
\hline Primer name & Sequence & Target/purpose \\
\hline tetB_for & CGCATTGGTAATTACGTTATTCGATG & Amplification of an $1101 \mathrm{bp}$ internal fragment of tet(B) \\
\hline tetB_rev & GCTAAACCAATAATCCAAATCCAGC & \\
\hline tetB_5'_out & CGTAATTACCAATGCGATCTITGTC & Inverse PCR amplification and/or sequencing of the region between the ends of the tet(B) gene \\
\hline tetB_3'_out & GTTAACCCCTCAAGCTCATGG & \\
\hline rep_5'_out & TTGCCATAAGACTAGAGATTTCCTG & Sequencing out from $5^{\prime}$ end of rep in p780 (multiple priming sites in other plasmids) \\
\hline rep_3'_out & TITAAGAGGGGAATATGGCAACAC & Sequencing out from $3^{\prime}$ end of rep in plasmids other than p780 where multiple priming sites are present \\
\hline 3362_node40_out & TTGCCATAAGACTAGAGATTTCCTG & Confirmation of correct joining of contigs and sequencing of the pM3362MDR plasmid \\
\hline 3362_node111_out & AGCCCAAAAAGAGCCGATAGG & \\
\hline 3362_node126_out & TTAATGTTCAGCAGAGGGGAGG & \\
\hline 3362_rep_5'_out & TAGAACTCTCATITCATCAAGCG & \\
\hline 3362_ISApl_5'_out & TCGTTGCACTTGGTTTGACAATTC & \\
\hline 3362_ISApl_3'_out & TGCCCTGTGCGAGTAAAATC & \\
\hline 780_rep_int_for & GGTITAGAGCCATCCATAACGG & Sequencing out from $3^{\prime}$ end of rep in p780 \\
\hline 780_node44_out & GCCATПTTACCTTCCTAATCTTCAG & Confirmation of correct joining of contigs and sequencing of the p780 plasmid \\
\hline
\end{tabular}

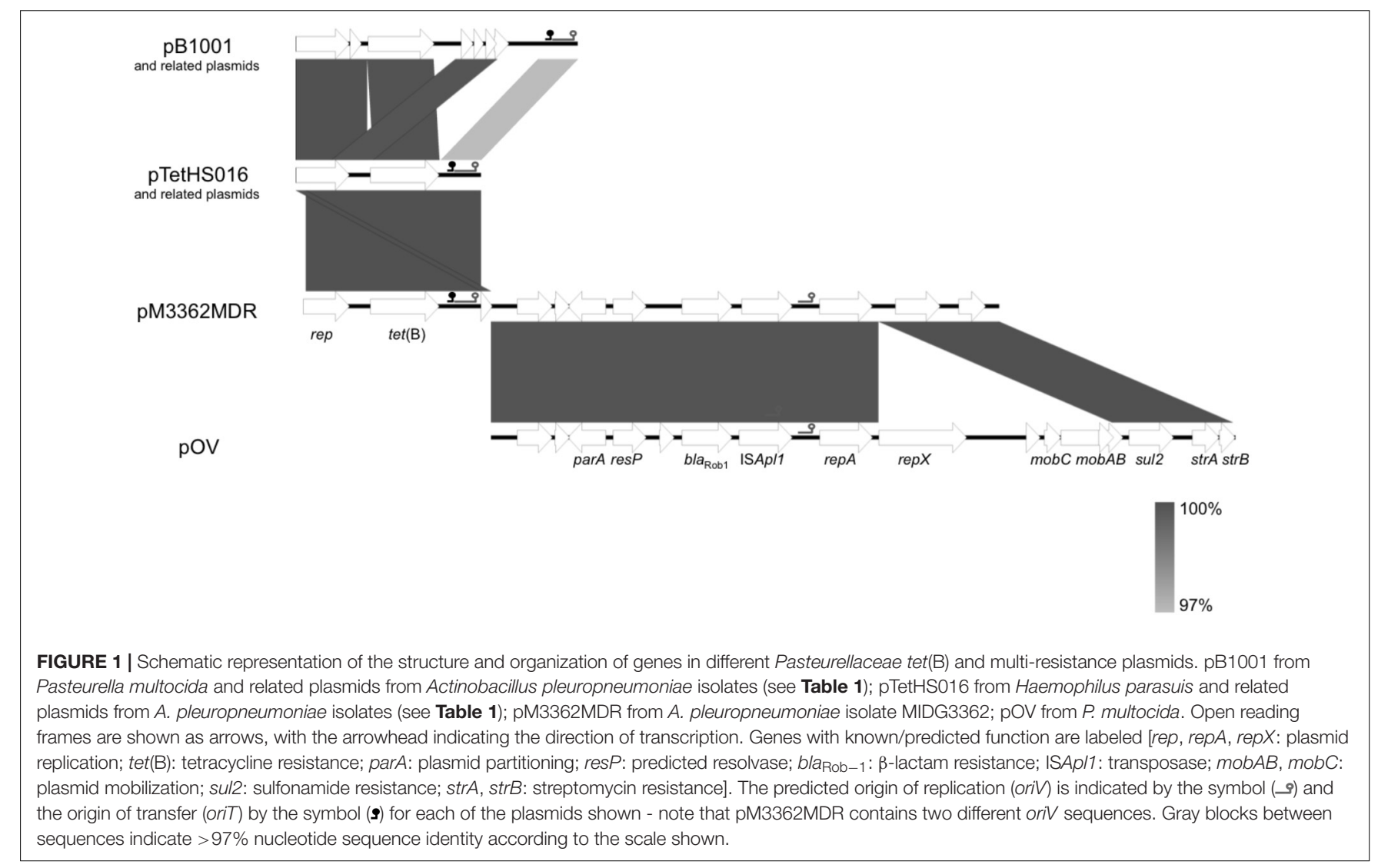

under the accession numbers MH457196 to MH457202, respectively.

\section{Electroporation of Plasmids Into Escherichia coli MFDpir}

Plasmids p780, pM2656Tet, and pM3362MDR were electroporated into the E. coli conjugal donor strain, MFDpir (Ferrieres et al., 2010). Transformants were selected on
Brain Heart Infusion (BHI) agar supplemented with $0.3 \mathrm{mM}$ diaminopimelic acid, containing $5 \mu \mathrm{g} / \mathrm{ml}$ tetracycline. The presence of plasmid in tetracycline resistant transformants was confirmed by PCR amplification using the tetB_for/tetB_rev primers (Table 2) prior to plasmid extraction, as above. Stability of the cloned plasmids in E. coli was assessed and compared to the endogenous plasmids in A. pleuropneumoniae as previously described (Moleres et al., 2015), with minor modifications. Briefly, following initial plating on selective agar, bacteria 

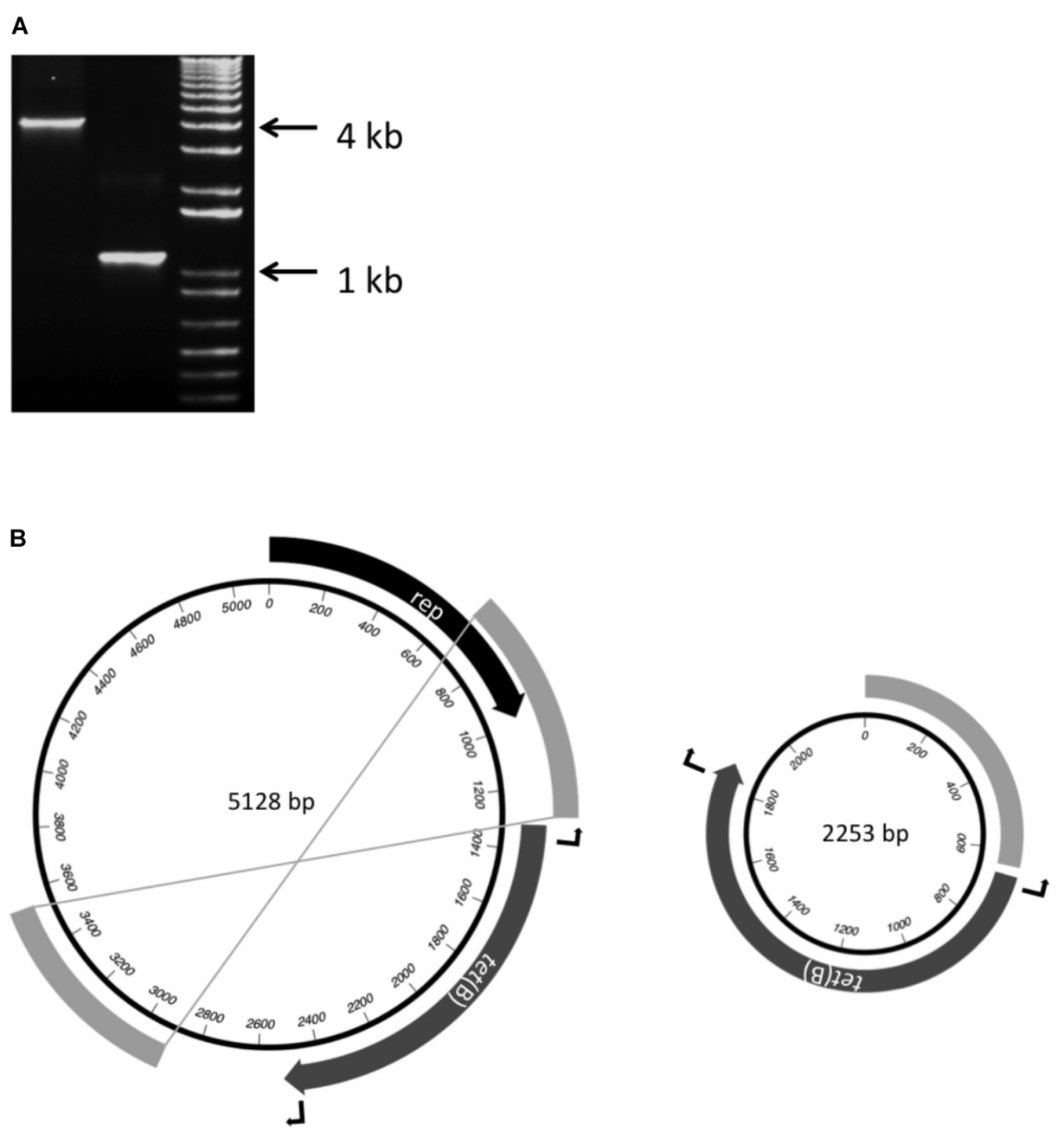

FIGURE 2 | Detection of two different sized amplicons (A) produced by inverse-PCR from the ends of the tet(B) gene using DNA extracted from the A. pleuropneumoniae isolate MV780. (B) Schematic representation of p780 (5128 bp) and a smaller plasmid (2253 bp) formed by recombination between the direct repeat sequences (shown as unlabelled gray bands) flanking the tet(B) gene, as indicated by the crossed lines joining the ends of the repeat sequences in p780. The locations of the outward-facing primers at the ends of the tet(B) gene are indicated by the bent arrows, and lengths of sequence between the primers in the respective plasmids correspond to the correspond to the 4 and $1.1 \mathrm{~kb}$ amplicons detected in (A).

were cultured in $10 \mathrm{ml}$ non-selective broth, which was serially passaged (1:100 dilution) eight times. Following the fourth and eighth passage, the stability of the plasmids was assessed by comparing the number of resistant $\mathrm{cfu} / \mathrm{ml}$ on selective agar, to total $\mathrm{cfu} / \mathrm{ml}$ on non-selective agar.

\section{Conjugal Transfer Experiments}

In order to investigate mobilization from the E. coli MFDpir clones containing plasmids p780, pM2656Tet, and pM3362MDR, we used the same plasmid-free, tetracycline susceptible clinical A. pleuropneumoniae isolates as previously used to demonstrate conjugal transfer of ICEApl1 and ICEApl2 (Bossé et al., 2016; Li et al., 2018), namely MIDG3376 (serovar 6), MIDG2465 (serovar 7), MIDG3217 (serovar 8), and MIDG3347 (serovar 12). Matings were performed as previously described (Bossé et al., 2009), with selection on BHI-NAD containing $2.5 \mu \mathrm{g} / \mathrm{ml}$ tetracycline. The conjugation experiments were performed twice on independent occasions, with MIDG2331 $\Delta$ ureC::nadV [carrying tet(B) in ICEApl1] as a positive control donor (Bossé et al., 2016).

\section{RESULTS AND DISCUSSION}

In each of the four Brazilian isolates, a 1206 bp tet(B) gene was on a 1611 bp contig that shares $100 \%$ identity with the rep and tet(B) sequences of pB1001 (Figure 1), a 5128 bp plasmid from Pasteurella multocida (San Millan et al., 2009). The remaining sequences of this plasmid were found on two further contigs (644 and 2231 or $2233 \mathrm{bp}$ ) in each isolate, with the $644 \mathrm{bp}$ contig (containing the last $329 \mathrm{bp}$ of rep) matching sequences both upstream and downstream of tet(B) in $\mathrm{pB} 1001$. When inverse PCR was used to determine the length of the sequence flanking the tet(B) gene, two products (circa 4 and $1.1 \mathrm{~kb}$, respectively; 


\section{$3^{\prime}$ end of strB from pOV

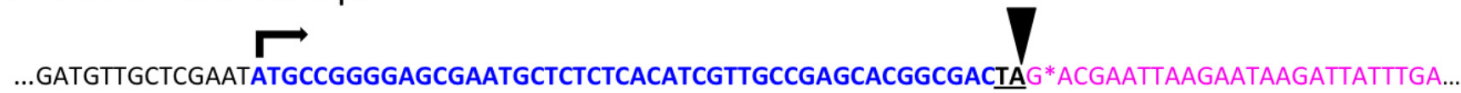

\section{5' end of rep from pTetHSO16}

ATGGCAAATGATTTAGTTGTAGTGAAAGCAAATAGTCTTATTGAAGCTAGTTACCGATTAAGTATTGATGAAATCCGTATTCTAGCTTTAACGATTGGAACAATGGATCCAAAATCAAAT CAGCAAATTTTGATTTACGGTAGCCGATTTTGTCCGTGAATTTCCTGAGATCAATATGGATAACGCTTATAAGCAAATTCAGGCGGCTATCAAACGGATTTATGA...

\section{5 ' end of rep from pM3362MDR}

ATGCCGgGGAGCGAATGCTCTCTCACATCGTTGCCGAgCACGGCGACIAACGCTTATAAGCAAATTCAGGCGGCTATCAAACGGATTTATGA...

\section{0 bp ORF downstream of oriV in pM3362MDR}

ATGGCAAATGATTTAGTTGTAGTGAAAGCAAATAGTCTTATTGAAGCTAGTTACCGATTAAGTATTGATGAAATCCGTATTCTAGCTTTAACGATTGGAACAATGGATCCAAAATCAAA TCAGCAAATTTTTGATTTTACGGTAGCCGATTTTGTCCGTGAATTTCCTGAGATCAATATGGATAGACGAATTAAGAATAAGATTATTTGA*

FIGURE 3 | Schematic representation of the site of insertion of the pTetHS016 plasmid sequence into a pOV-like plasmid during formation of pM3326MDR. The stop codon (TAG) of the strB gene in pOV is followed by an asterisk (*) indicating the end of the coding sequence. The insertion site of pTetHSO16 into a pOV-like plasmid is indicated by a downward triangle. The TA dinucleotide present in the stop codon of strB, and that in the rep gene of pTetHS016, at which point the plasmids recombine, is shown in bold and underlined in each of the sequences shown. During recombination, the $5^{\prime}$ end of the pTetHSO16 rep gene is displaced, forming the majority of the $210 \mathrm{bp}$ ORF found downstream of the oriV in pM3362MDR. An alternate $5^{\prime}$ end for the rep gene in pM3362MDR is supplied from within the $3^{\prime}$ end of the disrupted strB gene (shown in blue text), whereas the stop codon of the 210 bp ORF in pM3362MDR is derived from sequence following the end of the strB gene (sequence shown in pink text). The majority of the rep gene in pM3362MDR is identical to that in pTetHS016 (sequence shown in red italic text).

Figure 2A) were amplified, which when sequenced, indicated that the smaller amplicon was produced by deletion of $2875 \mathrm{bp}$ mediated by recombination between the direct repeat sequences flanking tet(B) (Figure 2B). This type of instability has been reported in other plasmids (Dianov et al., 1991; Oliveira et al., 2010). It is unclear whether the smaller plasmid, lacking the full-length rep gene, is capable of stable replication.

In eight United Kingdom isolates, a single contig was found to contain the entire sequence of a plasmid almost identical to pTetHS016 (Figure 1; accession KC818265), a 3366 bp plasmid from a United Kingdom Haemophilus parasuis isolate (Luan et al., 2013), encoding a 1257 bp tet(B) gene along with a 978 bp rep gene. In each case, direct repeat sequences at either end of the contig were detected that allowed closure into circular plasmids of similar sizes (Table 1). A further five isolates had sequences similar to pTetHS016 divided over two contigs (Table 1). Inverse PCR produced similar sized amplicons (circa $2.2 \mathrm{~kb}$ ) for sequences flanking the tet(B) gene in isolates with the pTetHS016-like sequences found on one or two contigs (Figure 1). Complete sequencing of pM2567Tet, pM2658Tet, pM3202Tet, and pM3233Tet confirmed the predicted sizes, with slight differences between plasmids seen in the intergenic regions.

Comparison of pB1001 and pTetHS016 (Figure 1) revealed that they share $95 \%$ identity over the majority of the smaller plasmid sequence, indicating that they likely evolved from the same origin. Differences around the $5^{\prime}$ and $3^{\prime}$ ends of tet(B) account for the variation in length of this gene between the plasmids, with a $110 \mathrm{bp}$ deletion in the pB1001-type replicons resulting in a start codon 16 residues into the protein encoded by the larger genes, and a single base deletion at the $3^{\prime}$ end resulting in an alternate stop codon. The tet(B) gene from the larger pB1001-type plasmids (as well as 350 bp downstream, not present in the smaller pTetHS016-type), shows 100\% identity with chromosomal and plasmid sequences from various species including E. coli, Salmonella enterica, and Shigella dysenteriae (e.g., accession numbers CP025254, CP022069, and CP026778), indicating a likely enterobacterial source, as has been shown for other resistance genes in Pasteurellaceae plasmids (Michael et al., 2018).

In the genome of one United Kingdom isolate, MIDG3362, a 1257 bp tet(B) gene was identified by ResFinder on a 6496 bp contig, 3051 bp of which shares 100\% identity with pTetHS016, and the remaining 3455 bp is $99 \%$ identical to sequences encoding repA, sul2, and str $A$ found in pOV (accession NC_019381), a 13,551 bp plasmid from from $P$. multocida, and a related set of small multi-resistance plasmids from "Actinobacillus porcitonsillarum" (Matter et al., 2008) ranging in size from 8751 to 13,425 bp (accession numbers AJ830711, AM748705, AJ830712, and AM748706). These plasmids all further carry the $\beta$-lactamase gene, $b l a_{\mathrm{Rob}-1}$. ResFinder results for MIDG3362 identified a 4820 bp contig carrying bla $a_{\text {Rob-1 }}$, of which 4485 bp is $99 \%$ identical to sequences in pOV, and the remaining $336 \mathrm{bp}$ is $100 \%$ identical with sequences in pTetHS016, with a 20 bp overlap, allowing the joining to 


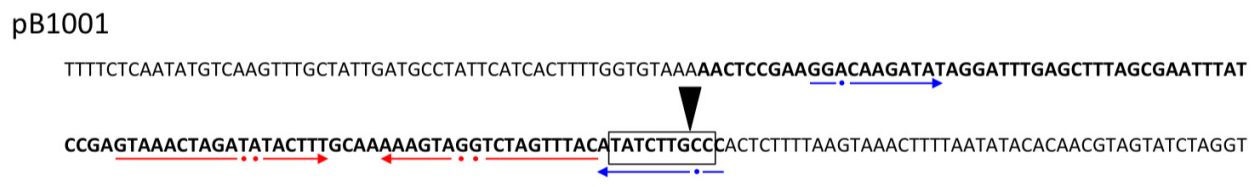

FIGURE 4 | Nucleotide sequences from plasmids pB1001, pTetHS016, and RP4 indicating the respective origin of transfer (oriT) regions in bold text, with the nic recognition site boxed and the site of cleavage indicated by a downward triangle. The major imperfect inverted repeat sequences in each are underlined with red arrows (broken, with dots indicating non-conserved bases), and the sequences of a second imperfect inverted repeat underlined with blue arrows (broken, with dots indicating non-conserved bases). In pB1001 (and related A. pleuropneumoniae plasmids - see Table 1), the oriT is located immediately upstream of the predicted origin of replication (oriV), an AT-rich region containing four iterons (direct repeats of the sequence TTATACGACTAGAAATTCCTG; indicated by the four contiguous arrows above the text) involved in binding of the Rep protein. In RP4, the oriT is located immediately upstream of the traJ gene sequence (for which only the $5^{\prime}$ end is shown encoded on the complement strand, with bases of the coding sequence in larger font, and with the respective amino acids shown below each codon). In $\mathrm{RP} 4$, the sequence downstream of the oriT does not contain the oriV, but rather leads to the divergently transcribed traK gene, following 225 bp of intergenic sequence.

pTetHS016 sequences on the tet(B) carrying contig. A further 1375 bp sharing $100 \%$ identity with sequences from pOV was found distributed over two additional contigs in the MIDG3362 genome. Overlapping sequences were identified between the ends of these two contigs, and those of the tet(B)- and the $b a_{\text {Rob-1-carrying contigs, allowing closure of a complete }}$ circular plasmid which was confirmed by PCR amplification and sequencing of products spanning the junctions. This $12666 \mathrm{bp}$ plasmid, pM3362MDR, contains an almost complete copy of the pTetHSO16 sequence (Figure 1). The pTetHS016 plasmid appears to have integrated seamlessly into the stop codon of the truncated $s t r B$ gene in plasmid pOV by a single cross-over event at a TA dinucleotide (Figure 3), resulting in disruption of the 978 bp rep gene, such that the $5^{\prime}$ end of the gene is part of a $210 \mathrm{bp}$ orf found downstream, whilst the majority of the rep gene is present as an $843 \mathrm{bp}$ orf upstream of tet (B), having acquired an alternate start codon from within the $3^{\prime}$ end of the strB gene of pOV. There are no apparent homologous sequences in the rep and $s t r B$ genes, thus integration appears to have been through illegitimate recombination. A $4255 \mathrm{bp}$ segment of pOV, spanning from rep $X$ through the mobCAB genes, is not present in pM3362MDR, where only a $199 \mathrm{bp}$ remnant of the mobA gene is present between repA and sul2. Deletion of this segment may explain why attempts to mobilize pM3362MDR from a conjugal donor strain into plasmid-free isolates of A. pleuropneumoniae were unsuccessful (see below). 


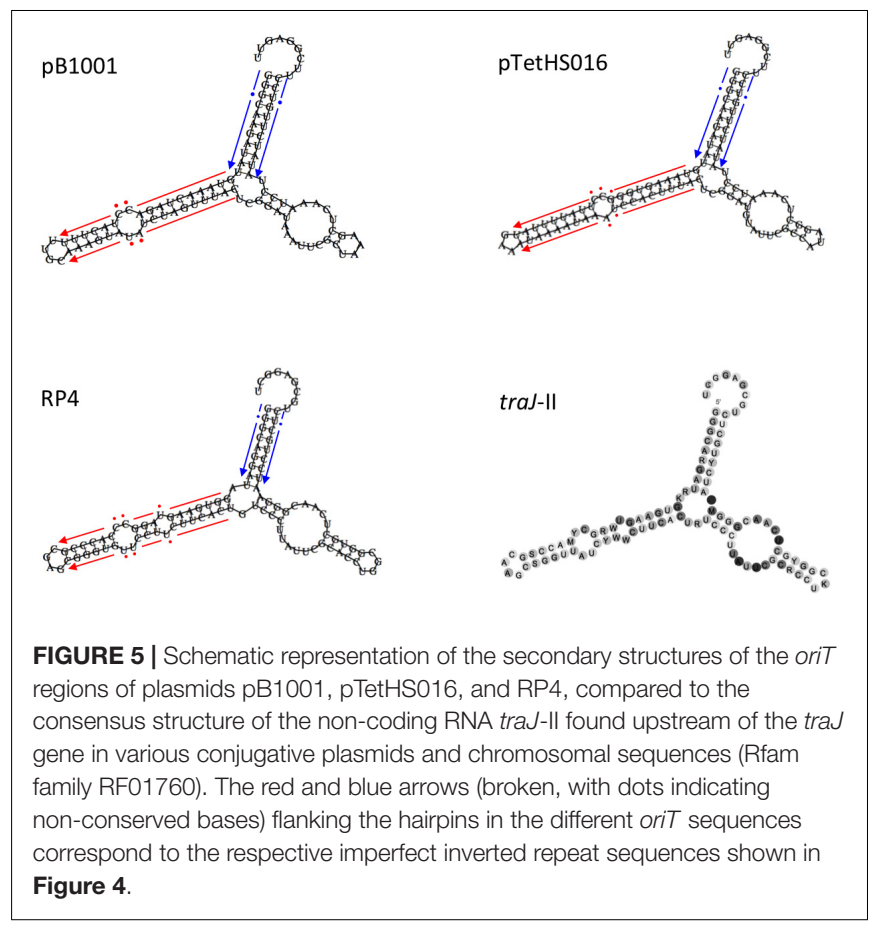

Replicons which have evolved to be stably maintained in Pasteurellaceae frequently contain resistance genes that appear to have been acquired from enterobacterial plasmids (AresArroyo et al., 2018; Michael et al., 2018). This is likely due to the fact that most Pasteurellaceae plasmids are capable of stable replication in E. coli, whereas the converse is rarely true, suggesting some differences in the origin (oriV) and/or proteins required for replication (Michael et al., 2018). In Pasteurellaceae species, many plasmids are ColE1-type replicons (related to, but distinct from those found in Enterobacteriaceae), which tend to carry the mobCAB mobilization genes, with the origin of transfer (oriT) as well as the oriV found upstream of mobC (Ares-Arroyo et al., 2018). Replication of ColE1-type plasmids relies on host factors; whereas plasmids carrying their own rep genes have cognate oriV sequences, typically AT-rich regions containing direct repeats (iterons) which specifically bind the Rep protein (Rajewska et al., 2012; Lilly and Camps, 2015; Wegrzyn et al., 2016). Both Rep-encoding and ColE1-type replicons have been described in Pasteurellaceae species, and some plasmids carry more than one oriV; for example, the pOV plasmid has both a ColE1 origin, and a RepA-specific oriV (discussed below).

The three different plasmids in this study, which were confirmed to stably replicate in both A. pleuropneumoniae and E. coli over eight passages in the absence of selection, have an AT-rich (74\%) region of 438-458 bp containing four contiguous direct repeats (iterons) of the $22 \mathrm{bp}$ sequence TTATACGACTAGAAATTTCCTG (shown for pB1001 and pTetHS016 in Figure 4). Although there is sequence variation between the AT-rich regions of the different plasmids, the iterons are identical, supporting that these are sites for binding the Rep protein encoded by all of these plasmids. In the smaller
pB1001- and pTetHS016-type plasmids, the predicted replication origin immediately precedes the $978 \mathrm{bp}$ rep gene, whereas in pM3362MDR, it is upstream of the $210 \mathrm{bp}$ orf containing the $5^{\prime}$ end of the rep gene, as discussed above. It is unclear if the truncated $843 \mathrm{bp}$ rep gene in $\mathrm{pM} 3362 \mathrm{MDR}$ is functional, as there is a second predicted oriV located upstream of the repA gene derived from the pOV-like replicon, which may be responsible for replication of this plasmid (Figure 1). This $292 \mathrm{bp}$ region has an AT-content of $69 \%$, and contains four contiguous direct repeats of the 22 bp sequence TTAAAACCCTACAGATTTACGG, which is likely the iteron specific for binding of the RepA protein. In support of this, an identical oriV was previously described in other plasmids encoding the same Rep protein (Matter et al., 2008).

Small mobilizable plasmids normally carry mob gene(s) encoding a relaxase (or multi-component relaxosome), which makes a single strand cut at the nic site of the oriT sequence, commonly found upstream (O'Brien et al., 2015). However, some mobilizable plasmids have been found that carry only a minimum oriT, requiring the relaxase to be supplied in trans (O’Brien et al., 2015). Relaxase proteins are specific for their cognate oriT sequences, and have been classified into six major families (i.e., MOB families $\mathrm{C}, \mathrm{F}, \mathrm{H}, \mathrm{P}, \mathrm{V}$, and Q), which can be further divided into subfamilies (GarcillánBarcia et al., 2009; Zrimec and Lapanje, 2018). Although the plasmids in this study do not encode known mob genes, they do all contain a 100-105 bp sequence (Figures 4, 5) located immediately upstream of the common ori $V$, containing regions of dyad symmetry that are characteristic of oriT sequences (Ziegelin et al., 1989; Pansegrau et al., 1990; O’Brien et al., 2015). These sequences are predicted to form secondary structures similar to the bioinformatically predicated sRNA traJ-II (traJII; Rfam family RF01760; Figure 5), found in the $5^{\prime}$ UTR of the traJ gene in various plasmid and chromosomal sequences (Weinberg et al., 2010). Indeed, the predicted traJ-II sequence in conjugative plasmid RK2/RP4 (accession number K00832.1) corresponds to that previously described as the oriT of this plasmid (Ziegelin et al., 1989; Pansegrau et al., 1990). Recently, a plasmid classification method was developed based on analysis of conserved structures in non-coding regions, which were shown to be highly conserved and discriminative for predicting the MOB family, even in the absence of encoded relaxases (Zrimec and Lapanje, 2018). Results obtained using this tool support our prediction that the oriT sequences identified in the plasmids in this study, like that of RP4, correspond to the MOBP family. However, as the largest family, MOBP includes numerous relaxases, some of which require accessory proteins for specific binding of their cognate oriT prior to cleavage of the nic site (Pansegrau and Lanka, 1991; Parker et al., 2005; Garcillán-Barcia et al., 2009; Zrimec and Lapanje, 2018).

In addition to having similar secondary structure, the oriT sequences of the plasmids in this study have predicted nic sites that share a high degree of identity with that of RP4, differing only at one base, however, the sequences of the major inverted repeat (found immediately upstream of the nic site) differ markedly (Figure 4). The right arm of this repeat region has been shown 
to provide specificity of the RP4 relaxosome via binding of the TraJ protein (Ziegelin et al., 1989; Pansegrau et al., 1990). We confirmed the inability of RP4-dependent conjugative transfer machinery, encoded by E. coli conjugal MFDpir (Ferrieres et al., 2010), to transfer representatives (pM2656Tet, pM3362MDR, and p780) of each of the three types of resistance plasmid in this study. Having been electroporated into MFDpir, the plasmids were shown to be capable of replication in this E. coli strain, as confirmed by PCR amplification of the encoded tet(B) gene from transformants, however, no transconjugants were obtained using any of the four plasmid-free A. pleuropneumoniae recipient strains. The control experiment demonstrated that these strains were successfully used as recipients for conjugal transfer of the tetracycline resistance integrative conjugative element, ICEApl1, with conjugation frequencies of $10^{-4}-10^{-5}$, both in this and the previous study (Bossé et al., 2016). It is possible that mobilization of the plasmids described in this study, homologs of which have been identified in different Pasteurellaceae species (suggesting horizontal transfer between them), requires specific conjugal transfer machinery that has yet to be identified. Various integrative and conjugative elements (ICEs) belonging to either the ICEHin1056 family (Mohd-Zain et al., 2004; Bossé et al., 2016), the SXT/R391 family (Li et al., 2018; Xu et al., 2018), or a set related ICEs including ICEPmu1 and ICEMh1 (Michael et al., 2012; Eidam et al., 2015), are present in different Pasteurellaceae species, but none have sequences similar to the predicted plasmid oriTs (or their major inverted repeats), suggesting the ICEencoded relaxases would not likely be capable of mobilizing these plasmids.

In summary, we have identified and characterized three related tetracycline resistance plasmids circulating in isolates of A. pleuropneumoniae in the United Kingdom and Brazil. Two are almost identical to plasmids pTetHS016 and pB1001, found in other members of the Pasteurellaceae (i.e., $H$. parasuis and $P$. multocida, respectively), that carry only the tet(B) and rep genes. The third, pM3362MDR, is a novel multiresistance plasmid, carrying $b l a_{\mathrm{Rob}-1}, s u l 2$, and $s t r A$ in addition to tet(B), and appears to have been derived from insertion of the pTetHS016 plasmid into pOV (from P. multocida), or a related plasmid. Co-existence of multiple plasmids in the

\section{REFERENCES}

Archambault, M., Harel, J., Gouré, J., Tremblay, Y. D. N., and Jacques, M. (2012). Antimicrobial susceptibilities and resistance genes of Canadian isolates of Actinobacillus pleuropneumoniae. Microb. Drug Resist. 18, 198-206. doi: 10. 1089/mdr.2011.0150

Ares-Arroyo, M., Bernabe-Balas, C., Santos-López, A., Baquero, M. R., Prasad, K. N., Cid, D., et al. (2018). PCR-based analysis of ColE1 plasmids in clinical isolates and metagenomic samples reveals their importance as gene capture platforms. Front. Microbiol. 9:177. doi: 10.3389/fmicb.2018.00469

Blanco, M., Gutiérrez-Martín, C. B., Rodríguez-Ferri, E. F., Roberts, M. C., and Navas, J. (2006). Distribution of tetracycline resistance genes in Actinobacillus pleuropneumoniae isolates from Spain. Antimicrob. Agents Chemother. 50, 702-708. doi: 10.1128/AAC.50.2.702-708.2006

Borriello, S. P. (2013). UK Veterinary Antibiotic Resistance and Sales Surveillance 2013. Available at: www.vmd.defra.gov.uk/pdf/varss.pdf

Bossé, J. T., Durham, A. L., Rycroft, A. N., Kroll, J. S., and Langford, P. R. (2009). New plasmid tools for genetic analysis of Actinobacillus pleuropneumoniae and same isolate has previously been reported for P. multocida and A. pleuropneumoniae (San Millan et al., 2009; Bossé et al., 2015), providing the opportunity for either homologous or illegitimate recombination. The presence of (nearly) identical plasmids in multiple Pasteurellaceae suggests horizontal transfer between these different species, which can share the same niche in the respiratory tract of pigs. Although a putative MOBP-related oriT was identified in all plasmids, we were unable to demonstrate mobilization using an E. coli donor strain expressing conjugation machinery specific for RP4-type plasmids. It is possible that these plasmids could be mobilized by cognate conjugation machinery, perhaps Pasteurellaceae-specific, which remains to be identified.

\section{AUTHOR CONTRIBUTIONS}

JB, PL, SW, GS, and DB conceived the study. SW provided UK clinical isolates. YHL, GS, JB, YWL, and CR produced the data. JB, YHL, GS, and RF analyzed the data. JB and YHL wrote the paper.

\section{FUNDING}

This work was supported by Fundação de Amparo à Pesquisa do Estado de Minas Gerais - FAPEMIG (APQ-02732-15); Conselho Nacional de Desenvolvimento Científico e Tecnológico CNPq; Coordenação de Aperfeiçoamento de Pessoal de Nível Superior - CAPES/PROEX, and the Biotechnology and Biological Sciences Research Council (BB/K021109/1, BB/G018553/1, and $\mathrm{BB} / \mathrm{M} 023052 / 1)$. JB was also funded by CONFAP - the United Kingdom Academies Fellowship (FAPEMIG - APQ00689-16). YHL was funded by San-Ming Project of Medicine in Shenzhen (No. SZSM201811071).

\section{ACKNOWLEDGMENTS}

We wish to thank Chris Teale from the APHA for his advice and input.

other Pasteurellaceae. Appl. Environ. Microbiol. 75, 6124-6131. doi: 10.1128/ AEM.00809-09

Bossé, J. T., Li, Y., Fernandez Crespo, R., Chaudhuri, R. R., Rogers, J., Holden, M. T. G., et al. (2016). ICEApl1, an integrative conjugative element related to ICEHin1056, identified in the pig pathogen Actinobacillus pleuropneumoniae. Front. Microbiol. 7:810. doi: 10.3389/fmicb.2016.00810

Bossé, J. T., Li, Y., Rogers, J., Fernandez Crespo, R., Li, Y., Chaudhuri, R. R., et al. (2017). Whole genome sequencing for surveillance of antimicrobial resistance in Actinobacillus pleuropneumoniae. Front. Microbiol. 8:311. doi: 10. 3389/fmicb.2017.00311

Bossé, J. T., Li, Y., Walker, S., Atherton, T., Fernandez Crespo, R., Williamson, S. M., et al. (2015). Identification of $d f r A 14$ in two distinct plasmids conferring trimethoprim resistance in Actinobacillus pleuropneumoniae. J. Antimicrob. Chemother. 70, 2217-2222. doi: 10.1093/jac/dkv121

Chopra, I., and Roberts, M. (2001). Tetracycline antibiotics: mode of action, applications, molecular biology, and epidemiology of bacterial resistance. Microbiol. Mol. Biol. Rev. 65, 232-260. doi: 10.1128/MMBR.65.2.232-260. 2001 
Dayao, D., Gibson, J. S., Blackall, P. J., and Turni, C. (2016). Antimicrobial resistance genes in Actinobacillus pleuropneumoniae, Haemophilus parasuis and Pasteurella multocida isolated from Australian pigs. Aust. Vet. J. 94, 227-231. doi: 10.1111/avj.12458

Dianov, G. L., Kuzminov, A. V., Mazin, A. V., and Salganik, R. I. (1991). Molecular mechanisms of deletion formation in Escherichia coli plasmids - I. Deletion formation mediated by long direct repeats. Mol. Gen. Genet. 228, 153-159. doi: 10.1007/BF00282460

Eidam, C., Poehlein, A., Leimbach, A., Michael, G. B., Kadlec, K., Liesegang, H., et al. (2015). Analysis and comparative genomics of ICEMh1, a novel integrative and conjugative element (ICE) of Mannheimia haemolytica. J. Antimicrob. Chemother. 70, 93-97. doi: 10.1093/jac/dku361

El Garch, F., de Jong, A., Simjee, S., Moyaert, H., Klein, U., Ludwig, C., et al. (2016). Monitoring of antimicrobial susceptibility of respiratory tract pathogens isolated from diseased cattle and pigs across Europe, 20092012: VetPath results. Vet. Microbiol. 194, 11-22. doi: 10.1016/j.vetmic.2016. 04.009

European Medicines Agency and European Surveillance of Veterinary Antimicrobial Consumption (2017). Sales of Veterinary Antimicrobial Agents in 30 European Countries in 2015. London: EMA.

Ferrieres, L., Hemery, G., Nham, T., Guerout, A. M., Mazel, D., Beloin, C., et al. (2010). Silent mischief: bacteriophage $\mathrm{Mu}$ insertions contaminate products of Escherichia coli random mutagenesis performed using suicidal transposon delivery plasmids mobilized by broad-host-range RP4 conjugative machinery. J. Bacteriol. 192, 6418-6427. doi: 10.1128/JB.00621-10

Garcia-Migura, L., Hendriksen, R. S., Fraile, L., and Aarestrup, F. M. (2014). Antimicrobial resistance of zoonotic and commensal bacteria in Europe: the missing link between consumption and resistance in veterinary medicine. Vet. Microbiol. 170, 1-9. doi: 10.1016/j.vetmic.2014.01.013

Garcillán-Barcia, M. P., Francia, M. V., and la Cruz de, F. (2009). The diversity of conjugative relaxases and its application in plasmid classification. FEMS Microbiol. Rev. 33, 657-687.

Li, Y., Li, Y., Fernandez Crespo, R., Leanse, L. G., Langford, P. R., and Bossé, J. T. (2018). Characterization of the Actinobacillus pleuropneumoniae SXTrelated integrative and conjugative element ICEApl2 and analysis of the encoded FloR protein: hydrophobic residues in transmembrane domains contribute dynamically to florfenicol and chloramphenicol efflux. J. Antimicrob. Chemother. 73, 57-65. doi: 10.1093/jac/dkx342

Lilly, J., and Camps, M. (2015). Mechanisms of theta plasmid replication. Microbiol. Spectr. 3:LAS-0029-2014. doi: 10.1128/microbiolspec.PLAS-0029-2014

Luan, S.-L., Chaudhuri, R. R., Peters, S. E., Mayho, M., Weinert, L. A., Crowther, S. A., et al. (2013). Generation of a Tn5 transposon library in Haemophilus parasuis and analysis by transposon-directed insertion-site sequencing (TraDIS). Vet. Microbiol. 166, 558-566. doi: 10.1016/j.vetmic.2013. 07.008

Matter, D., Rossano, A., Sieber, S., and Perreten, V. (2008). Small multidrug resistance plasmids in Actinobacillus porcitonsillarum. Plasmid 59, 144-152. doi: 10.1016/j.plasmid.2007.11.003

Michael, G. B., Bossé, J. T., and Schwarz, S. (2018). Antimicrobial resistance in Pasteurellaceae of veterinary origin. Microbiol. Spectr. 6:ARBA-0022-2017. doi: 10.1128/microbiolspec.ARBA-0022-2017

Michael, G. B., Kadlec, K., Sweeney, M. T., Brzuszkiewicz, E., Liesegang, H., Daniel, R., et al. (2012). ICEPmul, an integrative conjugative element (ICE) of Pasteurella multocida: structure and transfer. J. Antimicrob. Chemother. 67, 91-100. doi: 10.1093/jac/dkr411

Mohd-Zain, Z., Turner, S. L., Cerdeño-Tárraga, A. M., Lilley, A. K., Inzana, T. J., Duncan, A. J., et al. (2004). Transferable antibiotic resistance elements in Haemophilus influenzae share a common evolutionary origin with a diverse family of syntenic genomic islands. J. Bacteriol. 186, 8114-8122. doi: 10.1128/ JB.186.23.8114-8122.2004

Moleres, J., Santos-López, A., Lázaro, I., Labairu, J., Prat, C., Ardanuy, C., et al. (2015). Novel bla $a_{\mathrm{ROB}-1}$-bearing plasmid conferring resistance to $\beta$-Lactams in Haemophilus parasuis isolates from healthy weaning pigs. Appl. Environ. Microbiol. 81, 3255-3267. doi: 10.1128/AEM.03865-14
O’Brien, F. G., Yui Eto, K., Murphy, R. J. T., Fairhurst, H. M., Coombs, G. W., Grubb, W. B., et al. (2015). Origin-of-transfer sequences facilitate mobilisation of non-conjugative antimicrobial-resistance plasmids in Staphylococcus aureus. Nucleic Acids Res. 43, 7971-7983. doi: 10.1093/nar/gkv755

Oliveira, P. H., Prather, K. J., Prazeres, D. M. F., and Monteiro, G. A. (2010). Analysis of DNA repeats in bacterial plasmids reveals the potential for recurrent instability events. Appl. Microbiol. Biotechnol. 87, 2157-2167. doi: 10.1007/ s00253-010-2671-7

Pansegrau, W., Balzer, D., Kruft, V., Lurz, R., and Lanka, E. (1990). In vitro assembly of relaxosomes at the transfer origin of plasmid RP4. Proc. Natl. Acad. Sci. U.S.A. 87, 6555-6559.

Pansegrau, W., and Lanka, E. (1991). Common sequence motifs in DNA relaxases and nick regions from a variety of DNA transfer systems. Nucleic Acids Res. 19, 3455-3455. doi: 10.1093/nar/19.12.3455

Parker, C., Becker, E., Zhang, X., Jandle, S., and Meyer, R. (2005). Elements in the co-evolution of relaxases and their origins of transfer. Plasmid 53, 113-118. doi: 10.1016/j.plasmid.2004.12.007

Pereira, M. F., Rossi, C. C., de Carvalho, F. M., de Almeida, L. G. P., Souza, R. C., de Vasconcelos, A. T. R., et al. (2015). Draft genome sequences of six Actinobacillus pleuropneumoniae serotype 8 Brazilian clinical isolates: insight into new applications. Genome Announc. 3:e01585-14. doi: 10.1128/genomeA. 01585- 14

Rajewska, M., Wegrzyn, K., and Konieczny, I. (2012). AT-rich region and repeated sequences - the essential elements of replication origins of bacterial replicons. FEMS Microbiol. Rev. 36, 408-434. doi: 10.1111/j.1574-6976.2011.00300.x

San Millan, A., Escudero, J. A., Gutierrez, B., Hidalgo, L., Garcia, N., Llagostera, M., et al. (2009). Multiresistance in Pasteurella multocida is mediated by coexistence of small plasmids. Antimicrob. Agents Chemother. 53, 3399-3404. doi: 10.1128/ AAC.01522-08

Vanni, M., Merenda, M., Barigazzi, G., Garbarino, C., Luppi, A., Tognetti, R., et al. (2012). Antimicrobial resistance of Actinobacillus pleuropneumoniae isolated from swine. Vet. Microbiol. 156, 172-177. doi: 10.1016/j.vetmic.2011.10.022

Wegrzyn, K. E., Gross, M., Uciechowska, U., and Konieczny, I. (2016). Replisome assembly at bacterial chromosomes and iteron plasmids. Front. Mol. Biosci. 3:39. doi: 10.3389/fmolb.2016.00039

Weinberg, Z., Wang, J. X., Bogue, J., Yang, J., Corbino, K., Moy, R. H., et al. (2010). Comparative genomics reveals 104 candidate structured RNAs from bacteria, archaea, and their metagenomes. Genome Biol. 11:R31. doi: 10.1186/gb-201011-3-r31

Xu, J., Jia, H., Cui, G., Tong, H., Wei, J., Shao, D., et al. (2018). ICEAplChn1, a novel SXT/R391 integrative conjugative element (ICE), carrying multiple antibiotic resistance genes in Actinobacillus pleuropneumoniae. Vet. Microbiol. 220, 18-23. doi: 10.1016/j.vetmic.2018.05.002

Zankari, E., Hasman, H., Cosentino, S., Vestergaard, M., Rasmussen, S., Lund, O., et al. (2012). Identification of acquired antimicrobial resistance genes. J. Antimicrob. Chemother. 67, 2640-2644. doi: 10.1093/jac/dks261

Ziegelin, G., Fürste, J. P., and Lanka, E. (1989). TraJ protein of plasmid RP4 binds to a 19-base pair invert sequence repetition within the transfer origin. J. Biol. Chem. 264, 11989-11994.

Zrimec, J., and Lapanje, A. (2018). DNA structure at the plasmid origin-of-transfer indicates its potential transfer range. Sci. Rep. 8:1820. doi: 10.1038/s41598-01820157-y

Conflict of Interest Statement: The authors declare that the research was conducted in the absence of any commercial or financial relationships that could be construed as a potential conflict of interest.

Copyright (c) 2018 Li, da Silva, Li, Rossi, Fernandez Crespo, Williamson, Langford, Bazzolli and Bossé. This is an open-access article distributed under the terms of the Creative Commons Attribution License (CC BY). The use, distribution or reproduction in other forums is permitted, provided the original author(s) and the copyright owner(s) are credited and that the original publication in this journal is cited, in accordance with accepted academic practice. No use, distribution or reproduction is permitted which does not comply with these terms. 